BNL-101036-2013-CP

\title{
Precise lifetime measurements in light nuclei for benchmarking modern ab-initio nuclear structure models
}

\author{
C.J. Lister $^{1}$, , and E.A. McCutchan ${ }^{2}$ \\ ${ }^{1}$ Department of Physics, University of Massachusetts, Lowell, Lowell MA 01854 \\ ${ }^{2}$ National Nuclear Data Center, Brookhaven National Laboratory, Upton, NY 11973-5000, \\ USA \\ (Dated: June 4, 2013)

\begin{abstract}
Presented at the ND2013 International Conference on Nuclear Data for Science and Technology Sheraton New York Hotel \& Towers New York, NY 10019 USA
\end{abstract}

March 4-8, 2013

\author{
National Nuclear Data Center \\ Brookhaven National Laboratory \\ P.O. Box 5000 \\ Upton, NY 11973-5000 \\ www.nndc.bnl.gov
}

\section{U.S. Department of Energy Office of Science, Office of Nuclear Physics}

\begin{abstract}
Notice: This manuscript has been authored by employees of Brookhaven Science Associates, LLC under Contract No. DE-AC02-98CH10886 with the U.S. Department of Energy. The publisher by accepting the manuscript for publication acknowledges that the United States Government retains a non-exclusive, paid-up, irrevocable, world-wide license to publish or reproduce the published form of this manuscript, or allow others to do so, for United States Government purposes.
\end{abstract}

This preprint is intended for publication in a journal or proceedings. Since changes may be made before publication, it may not be cited or reproduced without the author's permission. 


\section{DISCLAIMER}

This report was prepared as an account of work sponsored by an agency of the United States Government. Neither the United States Government nor any agency thereof, nor any of their employees, nor any of their contractors, subcontractors, or their employees, makes any warranty, express or implied, or assumes any legal liability or responsibility for the accuracy, completeness, or any third party's use or the results of such use of any information, apparatus, product, or process disclosed, or represents that its use would not infringe privately owned rights. Reference herein to any specific commercial product, process, or service by trade name, trademark, manufacturer, or otherwise, does not necessarily constitute or imply its endorsement, recommendation, or favoring by the United States Government or any agency thereof or its contractors or subcontractors. The views and opinions of authors expressed herein do not necessarily state or reflect those of the United States Government or any agency thereof. 


\title{
Precise lifetime measurements in light nuclei for benchmarking modern ab-initio nuclear structure models
}

\author{
C.J. Lister ${ }^{1, *}$ and E.A. McCutchan ${ }^{2}$ \\ ${ }^{1}$ Department of Physics, University of Massachusetts, Lowell, Lowell MA 01854 \\ ${ }^{2}$ National Nuclear Data Center, Brookhaven National Laboratory, Upton, NY 11973-5000, USA
}

(Dated: June 4, 2013)

\begin{abstract}
A new generation of ab-initio calculations, based on realistic two- and three-body forces, is having a profound impact on our view of how nuclei work. To improve the numerical methods, and the parameterization of 3-body forces, new precise data are needed. Electromagnetic transitions are very sensitive to the dynamics which drive mixing between configurations. We have made a series of precise $(<3 \%)$ measurements of electromagnetic transitions in the $A=10$ nuclei ${ }^{10} \mathrm{C}$ and ${ }^{10} \mathrm{Be}$ by using the Doppler Shift Attenuation method carefully. Many interesting features can be reproduced including the strong $\alpha$ clustering. New measurements on ${ }^{8} \mathrm{Be}$ and ${ }^{12} \mathrm{Be}$ highlight the interplay between the alpha clusters and their valence neutrons.
\end{abstract}

\section{INTRODUCTION}

Ideally, one would like to describe all nuclear systems from the most fundamental level of quarks and gluons. However, the span of energy and length scales and the complexity of the force appears to make a single theory intractable in the foreseeable future. The central theme of contemporary nuclear structure physics is to produce a set of closely-linked models which can build up starting from quarks and gluons, to nucleons and the inter-nucleon forces, then using these forces to describe light nuclei and the rise of the mean field and effective forces, and hence to configuration-mixing models for middle-mass nuclei, and finally to density functional theories to describe the heaviest nuclei with many hundreds of nucleons. The key issue in this hierarchy is to retain the important physics at each level of truncation and avoid arbitrary renormalizations. As we move from the well-known nuclei along the valley of stability, to the drip lines and up to the very heaviest nuclei, we need to better understand the evolution of nuclear matter as it becomes more and more exotic. This is especially true for nuclei with large neutron excesses, where our predictive power of nuclear structure is poorest, but where most heavy nucleosynthesis occurs. The lessons we can learn from the nuclei that we can synthesize on earth provide the only guide we have to some of the most exotic nuclear material that we think exists in the universe, for example in the crusts of neutron stars. Paradoxically then, studying the lightest nuclei very care-

\footnotetext{
* Corresponding author:

ChristopherLister@uml.edu
}

fully can teach us a great deal about the heaviest nuclear systems in the cosmos.

A wide variety of new nuclear models are forging the links between bare nuclear forces and nuclear structure. In this paper we will concentrate on testing the Green's Function Monte Carlo (GFMC) approach of Pieper and Wiringa $[1,2]$, as it is these calculations which motivated our experimental campaigns. However, other approaches, including "No Core" shell models [3, 4], effective field theories [5], lattice-based approaches [6, 7] and cluster models $[8,9]$ are similarly motivated. To really challenge these models, a new generation of precise experiments has started, especially in light nuclei. Mass measurements $[10,11]$ and RMS radii $[12-14]$ test the overall binding and topology of the wave functions, while sub-barrier fusion [15, 16], knockout [17] and transfer reactions [18] are all sensitive to the radial parts of the wave function. In this paper, we will discuss the measurement of precise electromagnetic transitions [19, 20] as a useful additional tool for investigating how different nuclear configurations mix. In all these tests, there is an interesting interplay of improving the actual computational methods and convergence, and developing improved nuclear forces. The computations involve massively parallel computing and most of them were simply not feasible until this century.

The approach of Pieper and Wiringa starts from the Argonne V18 two-body force [21], which is quite well known and is constrained by thousands of measurements, so is kept fixed. Constructing a proper nuclear wave function and minimizing its energy with just a two-body never produces enough binding energy. A key early finding was that three-body and higher correlations are essential to reproduce the known binding energy of light systems [22]. This presents a challenge, as these higher correlations 
are far less well understood and constrained by measurements. Thus, some unknown parameters must be introduced and fitted. However, over the last two decades the Illinois school has developed a series of three body interactions which capture more and more of the essential physics [23]. These correlations contribute $\sim 10 \%$ of the binding energy, but are responsible for half of the effective spin-orbit and tensor forces. They have strong isospin dependence, so are crucial for understanding exotic nuclei with large neutron excesses. They are most influential when the nucleons are close together, so are very significant in "clusters" of nucleons. Consequently, a good place to further investigate three-body forces is in the most clustered nuclei, like helium, beryllium, and carbon isotopes. This was the reason for choosing the beryllium isotopes ${ }^{8,10,12} \mathrm{Be}$ for our particular attention. Beryllium at heart is a di-alpha cluster, which can tumble end-overend, and emit electromagnetic radiation. The rate of radiation is directly related to the size of the radiating antenna, that is, the alpha-cluster spacing, so a precise measurement of the decay rate gives the state lifetime, and directly reveals the cluster spacing, the influence of "valence" neutrons, and the mixing of "tumbling" with other configurations. The experimental challenge is refining precision from the level of tens of percent achieved in the 1960's to the few percent level in the transitional matrix elements.

\section{EXPERIMENTS ON BERYLLIUM ISOTOPES}

The state of interest, the "tumbling" state, is known to have a lifetime of $\sim 200$ fs so is amenable to the Doppler Shift Attenuation Method (DSAM) [24]. To employ this method, the excited state of interest should be formed in a nuclear reaction in which the parent nucleus is produced with a high and well determined velocity. When the state decays by gamma emission, the radiation observed in the laboratory is then Doppler Shifted. The measured energy of the gamma ray depends on the intrinsic energy difference between the states under study, on the detection angle of the gamma rays and on the parent velocity. If the state decayed instantly, the velocity inferred from the Doppler shift would match the production velocity (which can be calculated from reaction kinematics). However, if the nucleus is de-accelerating, then the Doppler Shift is reduced. Only slowing the nuclei in solid materials can achieve the degree of de-acceleration needed for studying these short-lived states. The reduction of shift depends on a convolution of the slowing history and on the lifetime of the state. If the slowing history is known, then the lifetime can be inferred. Our experimental method is simply to do this measurement with careful attention to detail.

- We use 2-body reactions, in inverse kinematics and with large Q-values to make the nuclei recoil fast, thus increasing the size of Doppler shifts. In our ${ }^{10} \mathrm{C}$ project $[20]$ the initial recoil velocity was $\sim 12 \%$ c.
- We use very thin production targets to make the initial ensemble have uniform velocity.

- We detect the recoiling nucleus in a spectrometer, the Argonne Fragment Mass Analyzer (FMA) [25] in a small recoil cone near zero degrees. This helps determine the recoil-gamma opening angle, removes backgrounds from other reactions, and allows us to select reactions that directly populates our state of interest.

- We detect the gamma rays at 16 different angles, using the national gamma ray facility Gammasphere [26]. This allows many cross-checks and tests for systematic uncertainties. For example, we can detect a mis-alignment of the target of $<1 \mathrm{~mm}$.

- We use different slowing materials, like aluminum, copper, tantalum, and gold, and targets of different thicknesses, to cross check our modeling of slowing and the stopping powers $[27,28]$. In the 1960's, the paucity of data on stopping of ions in materials was the leading limitation of this technique, but vast progress has been made both in modeling and measurement, so in the velocity regime we use, the stopping powers are known at the few percent level.

In short, these studies revealed several things. Technically, very precise DSAM is possible. Figure 1 illustrates this precision using data from our ${ }^{10} \mathrm{C}$ measurement. The average velocity of the nucleus when the $\gamma$-ray is emitted can be measured to very high precision $(<1 \%)$, as given in Fig. 1 (top). In turn, the data are very sensitive to the lifetime of the level, as shown in Fig. 1 (bottom). We believe the current limitation is targetry: knowing exactly the composition and thickness of the production layers is a key. We worry about non-uniformity of thickness on the less than $\mathrm{mm}$ scale, i.e. across the beam spot. Another concern is deterioration of the targets under bombardment: do they retain their thickness? These things can be monitored by moving the beam, changing the target often, monitoring yield with time, but they are the leading uncertainties. Perhaps nano-technology will come to the rescue and allow fabrication of targets by atomic layer deposition. In physics, the GFMC proved reliable in predicting the overall topology of the antenna; that is the total quadrupole strength. We measured the sum of the $B(E 2)$ values from the first and second $J^{\pi}=2^{+}$states in ${ }^{10} \mathrm{Be}$ as $9.3(3) e^{2} \mathrm{fm}^{4}$ and the calculation predicted $10.5(3)$ $e^{2} \mathrm{fm}^{4}$ [19]. The projection of the density distribution of these wave functions clearly show both the two distinctly separated alpha clusters and the near-spherical cloud of neutrons. What was not quite so good is the configuration mixing. In nature, the first $J^{\pi}=2^{+}$state comes from "tumbling" of the di-alpha cluster, that is, it is the first member of the ground-state band, while the second $J^{\pi}=2^{+}$state arises from re-coupling the two valence neutrons from $\mathrm{J}=0$ to $\mathrm{J}=2$. In nature, it turns out that these modes are very decoupled, with the neutron re-coupled state radiating 80 times slower. Nature clearly likes this 


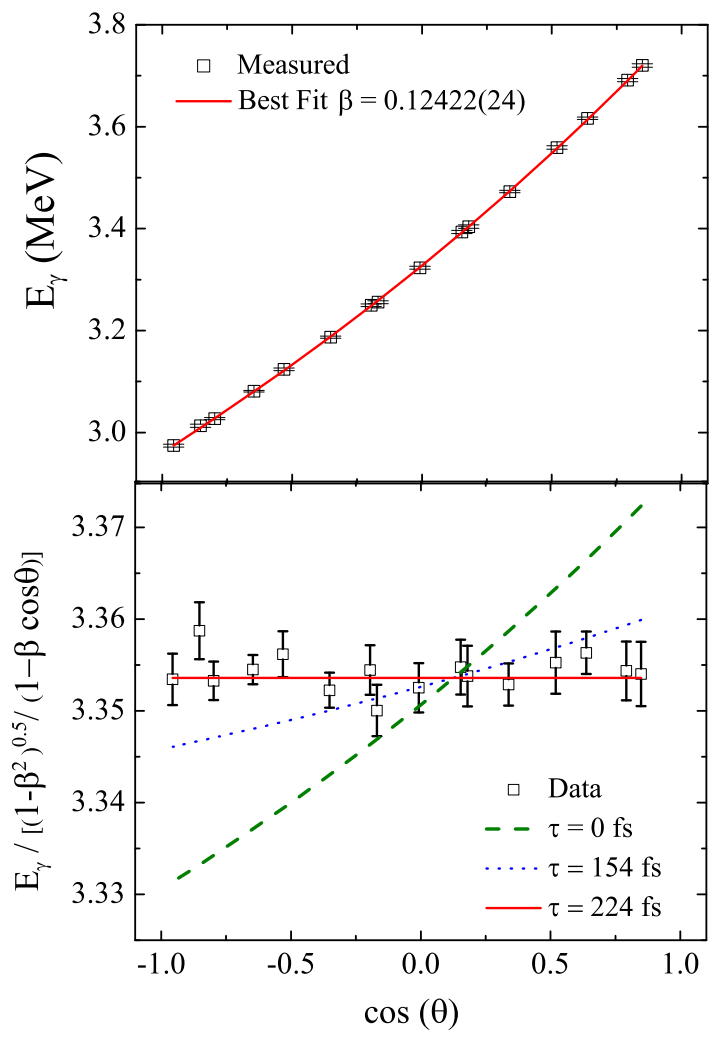

FIG. 1. (Color online) An illustration of a contemporary high precision DSAM measurement, showing (top) the Doppler shift of the first excited state in ${ }^{10} \mathrm{C}$ and (bottom) the sensitivity of the shift to the lifetime of the state.

symmetry. In GFMC, with all its sophistication, there are many, many, configurations involved in describing the di-alpha cluster, and the forces that we use converge best on a wave function which has more configuration mixing between the two modes than actually happens in nature. This is subtle, as a tiny (few percent) impurity of the fast-radiating tumbling wave function into the almost non-radiating neutron state causes a drastic enhancement of radiation from the latter. A key driver of this configuration mixing is the three-body force, so this mixing may be one of the most sensitive measurements we have for better constraining the interplay between the Feynman diagrams which describe the three-body interaction.

Of course, all these things must be reviewed from a distance. Adjusting some parameters to make an exact fit for the ${ }^{10} \mathrm{Be} B(E 2)$ values would be possible, but against the spirit of this project and probably not very informative in the long run. Consequently, it becomes important to see if similar effects are found in neighboring and closely related nuclei. One key experiment is measuring the analogous $B(E 2)$ strength in neighboring ${ }^{8} \mathrm{Be}$, the unbound di-alpha cluster. This difficult project has been undertaken by a group in Mumbai, India [29, 30]. It is a very challenging experiment: the ground state of ${ }^{8} \mathrm{Be}$

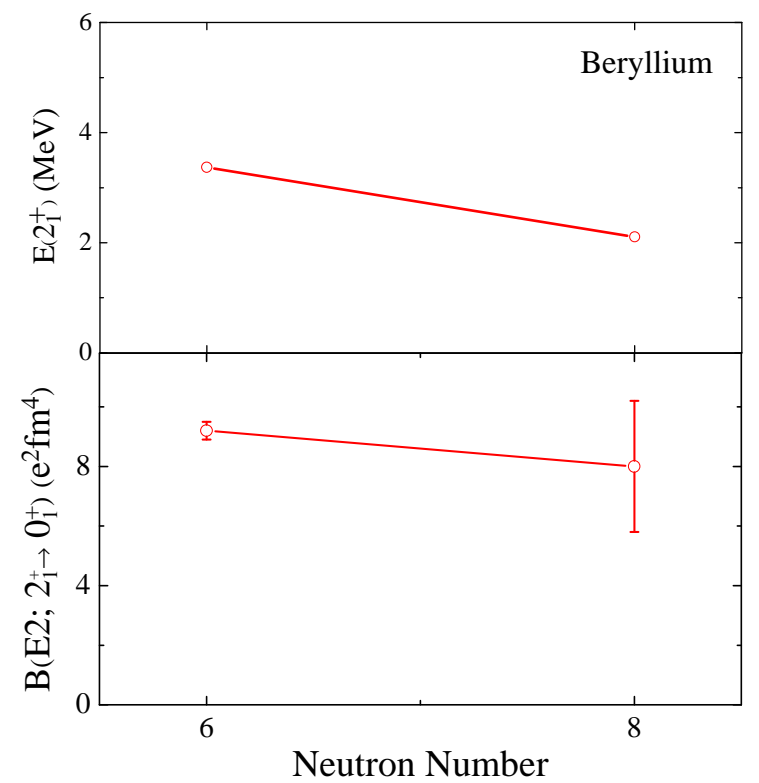

FIG. 2. (Color online) The systematics of level energies and electromagnetic transition strengths in beryllium isotopes. It is hard to reconcile these trends with conventional nuclear models.

is unbound by $\sim 90 \mathrm{keV}$ and has a lifetime of $\sim 10^{-16}$ seconds, and all the other states are broad unbound resonances. In this study the focus is on the $J^{\pi}=4^{+} \rightarrow 2^{+}$ tumbling transition. The parent state lies at $11.35 \mathrm{MeV}$ but is $3.5 \mathrm{MeV}$ wide. The key measurement is to determine the radiative branching ratio, that is, the ratio of electromagnetic decay to $\alpha-\alpha$ breakup. This gamma branch is at the $10^{-6}$ level, that is a radiative capture cross section of a few hundred nanobarns which has now been determined to $<10 \%$. Surprisingly, it appears that the topology in ${ }^{8} \mathrm{Be}$ excited states is very similar to that of ${ }^{10} \mathrm{Be}$, despite their frail unbound nature, and the fact that the $\alpha$ - $\alpha$ cluster in the $\mathrm{J}=4$ state barely survives for a whole rotation.

On the other side of ${ }^{10} \mathrm{Be}$, in more neutron-rich nuclei, one may ask if extra neutrons have much influence on the di-alpha core. ${ }^{12} \mathrm{Be}$ with two extra neutrons is very interesting for a variety of reasons. As the neutrons in ${ }^{10} \mathrm{Be}$ seem quite inert, one may naively expect ${ }^{12} \mathrm{Be}$ to be very similar in structure and radiation pattern. In fact nature is not that simple, the first excited state falls from 3368 $\mathrm{keV}$ to $2102 \mathrm{keV}$ [31] (see Fig. 2 (top)), which for a nuclear liquid drop would suggest a big increase in inertia, usually associated with elongation. Alternatively, as ${ }^{12} \mathrm{Be}$ has $N=8$ the "magic" number as found in ${ }^{16} \mathrm{O}$, one may anticipate a collapse of the alpha clustering and a return to sphericity and single particle-like $B(E 2)$ strengths. More exotically [32, 33], it has been suggested that the cloud of poorly bound neutrons can form Van-der-Waalslike molecular bonds between the alpha-clusters, leading to enhanced separation, especially in states with small binding energy. For the GFMC studies a calculation for 
${ }^{12} \mathrm{Be}$ really is a cutting edge project, as it involves several particles in the sd-shell model space and thus a big increase in the number of basis states involved. The theoretical predictions have very different radiation patterns, so again lifetime measurements become the key. A pioneering attempt at measuring the lifetime of this $\mathrm{J}=2$ state of ${ }^{12}$ Be in Japan [34] suggests the tumbling $B(E 2)$ is similar to ${ }^{10} \mathrm{Be}$ albeit with large uncertainty as shown in Fig. 2 (bottom). Thus, the wide ranging theoretical interpretations cannot currently be ruled out based on the $B(E 2)$ information. The recently-measured RMS radius of ${ }^{12} \mathrm{Be}[14]$ suggests the ground state is at least consistent with a di-alpha cluster slightly larger than that in ${ }^{10} \mathrm{Be}$, and certainly not a spherical $N=8$ configuration nor a very elongated di-alpha shape. In December 2012, at the NSCL facility at Michigan State University, we have repeated the Japanese study of ${ }^{12}$ Be using inelastic scattering, but with the state-of-the art tracking gamma ray detector GRETINA [35] and the S800 spectrometer [36] to measure and reconstruct the swiftly moving ions at 55 $\mathrm{MeV} / \mathrm{u}(\sim 30 \% \mathrm{v} / \mathrm{c})$ and adopting some of the experimen- tal techniques from our ${ }^{10} \mathrm{Be}$ experiment. We don't have results yet, but the data we collected were of the highest quality and we anticipate a result which will resolve the question of the ${ }^{12} \mathrm{Be}$ topology.

\section{CONCLUSIONS}

These are exciting times in the world of light nuclei and their structure. New precise measurements and abinitio theories are turning qualitative understanding into quantitative. The days of effective forces and effective charges which used to be required to reproduce nuclear structure and electromagnetic decays are disappearing as we move towards a more profound understanding. Real predictive power is improving [37]. The current challenge is to package and export this wisdom in a way that can be transported to Configuration Interaction models and Density Functionals which can then be used to reliably predict all nuclear systems.
[1] Steven C. Pieper, R. B. Wiringa, ANN. REV. NUCL. PART. SCI. 51, 53 (2001).

[2] R.B. Wiringa, AIP CONF. PROC. 1128, 1 (2009).

[3] E. Caurier, P. Navrátil, W. E. Ormand, J. P. Vary, PHYS. REV. C 66, 024314 (2002).

[4] B.R. Barrett, P. Navratil, J.P. Vary, PROG. PART. NUCL. PHYS. 69, 131 (2013).

[5] E. Epelbaum, H.-W. Hammer, U.-G. Meissner, REV. MOD. PHYS. 81, 1773 (2009).

[6] D. Lee et al., PHYS. REV. C 70, 104007 (2004).

[7] U.-G. Meissner, Proceedings of the Rutherford Centennial Conference, Manchester UK, IOP Publishing Conference Seris 381, 012017 (2012).

[8] T. Neff, H. Feldmeier, NUCL. PHYS. A 738, 357 (2004).

[9] Y. Kanada En'yo, H. Horiuchi, PHYS. REV. C 55, 2860 (1997).

[10] V.L. Ryjkov et al., PHYS. REV. LETT. 101, 012501 (2008).

[11] M. Broudeur et al., HYPERFINE INTERACTIONS 199, 167 (2011).

[12] P. Mueller et al., PHYS. REV. LETT. 99, 252501 (2007).

[13] W. Nörtershäuser et al., PHYS. REV. LETT. 102, 062503 (2009).

[14] A. Kreiger et al., PHYS. REV. LETT. 108, 142501 (2012).

[15] T. Neff, H. Feldmeier, K. Langanke, IOP CONF. SER. 337, 012055 (2012).

[16] K.M. Nollet, S.C. Pieper, R.B. Wiringa, J. Carlson, G.M. Hale, PHYS. REV. LETT. 99, 022502 (2007).

[17] G. F. Grinyer et al., PHYS. REV. LETT. 106, 162502 (2011).
[18] A. H. Wuosmaa et al., PHYS. REV. LETT. 94, 082502 (2005)

[19] E. A. McCutchan et al., PHYS. REV. LETT. 103, 192501 (2009).

[20] E.A. McCutchan et al., PHYS. REV. C 86, 014312 (2012).

[21] R.B. Wiring, V.G.J. Stocks, R. Schiavilla, PHYS. REV. C 51, 38 (1995).

[22] J. Fujita, H. Miyazawa, PROC. THEOR. PHYS. 17, 360 (1957).

[23] S. Pieper, AIP CONFERENCE SERIES 1011, 143 (2008).

[24] A.Z. Schwartzschild and E.K. Warburton, ANN. REV. NUCL. SCI. 18, 265 (1968).

[25] C. N. Davids et al., NUCL. INSTR. METH. PHYS. RES. B 70, 358 (1992).

[26] I-Yang Lee, NUCL. PHYS. A 520, 641c (1990).

[27] J. F. Ziegler, J. P. Biersack, and M. D. Ziegler, SRIM:The Stopping of Ions in Matter, Lulu Press, Morrisville, North Carolina (2008) http: \\www.srim.org

[28] H. Paul and A. Schinner, NUCL. INSTR. METH. PHYS. RES. B 195, 166 (2002).

[29] V.M. Datar et al., PHYS. REV. LETT. 94, 122502 (2005).

[30] V.M. Datar et al., - to be published.

[31] H.T. Fortune, G.-B. Liu, D.E. Alburger, PHYS. REV. C 50, 1355 (1994).

[32] M. Freer et al., PHYS. REV. LETT. 82, 1383 (1999).

[33] A. Saito et al., NUCL. PHYS. A 738, 337 (2004).

[34] N. Imai et al., PHYS. LETT. B 673, 179 (2009).

[35] I-Yang Lee, NUCL. PHYS. A 834, 743c (2010).

[36] D. Bazin et al., NUCL. INSTR. METH. B 204, 629 (2004).

[37] S.C. Pieper, PHYS. REV. LETT. 90, 252501 (2003). 